\title{
Automatic Ductile Damage Detection in Microalloyed Fine-Grain Steels
}

\author{
W. Rehbach*, U. Prahl**, C. Kuckertz**, W. Bleck** \\ * Central Facility for Electron Microscopy, Aachen University, 52056, GERMANY, \\ **Department of Ferrous Metallurgy, Aachen University, 52056, GERMANY
}

Ductile damage is caused by a heterogeneous stress strain distribution on the microscale due to the two phase morphology of brittle non-metallic inclusions distributed in a metallic matrix. Failure in these materials is caused by void formation due to particle cracking or interface debonding with subsequent void growth due to plastic incompressibility of the ductile matrix. At the end, micro crack formation occurs by spontaneous coalescence of two neighbouring voids.

In the present study ductile damage is investigated by means of microstructural observations. The material under investigation is a microalloyed thermomechanically treated steel S460M. This construction steel consists of sparse distributed inclusions (Al, Ca, Si, Mg-oxides) in a fine-grain microstructure of ferrite-bainite-perlite. Figure 1.b shows a SEM picture of S460M where the nonmetallic brittle inclusions can be found as dark points in a light ductile matrix. It must be pointed out that voids appear as dark points, too. Optically, no difference can be found between voids and inclusions at this magnification scale.

In order to determine the evolution of ductile damage during quasistatic loading several cylindrical notched tensile specimen (B8X40, notch radius $2 \mathrm{~mm}$, notch depth $1.5 \mathrm{~mm}$ ) are loaded up to different load levels. After interruption of the tensile test, the specimen were cut in longitudinal direction and the symmetry plane was polished and coated with an $8 \mathrm{~nm}$ C-layer. The specimens were investigated by means of SEM in combination of a X-EDS analysis, which is automatic controlled by an image analysis software. In the notch domain of the loaded specimen (figure 1.a) a grey scale analysis is performed on each backscattered-electron image in order to detect objects (voids or inclusions) and to measure the global position, the size and the ellipticity (figure 1.b). At each dark object an EDSspectrum is recorded and stored automatically. In figure 1.c $+d$, the spectra are shown of a Si-particle and a Ca-particle, respectively.

In the analysis, the values of $\mathrm{Al}-, \mathrm{Si}-$ and $\mathrm{Ca}-\mathrm{K}_{\alpha}$ are used to distinguish inclusions from voids, where a void is assumed if the $\mathrm{Al}$ counts are lower than 150 and the $\mathrm{Si}$ as well as the Ca counts are lower than 200. Furthermore, objects with an area greater than $100 \mu \mathrm{m}^{2}$ are assumed to be caused by artefacts, mainly in the notch domain due to embedding material, and thus, they are deleted. After this, the investigated area is divided into subareas using the Voronoi-algorithm with the detected objects as nuclei. Thus, for each object a local area can be assigned which is assumed to carry its deformation. The quotient of the objects area divided by the assigned Voronoi cell area is called the local area density of the object. In figure 2, this approach is shown for a $2 \times 2 \mathrm{~mm}^{2}$ investigation area for voids and inclusions, respectively.

In figure 3, the spectrum of voids as well as of inclusions is given using the size, the distance to the next neighbour and the ellipticity. These results can be used as an input for damage evolution investigations depending from the global load stage.

[1] The financial support of the Deutsche Forschungsgemeinschaft (DFG) within the collaborative research centre (SFB) 370 "Integrated Modelling of Materials" is gratefully acknowledged. 


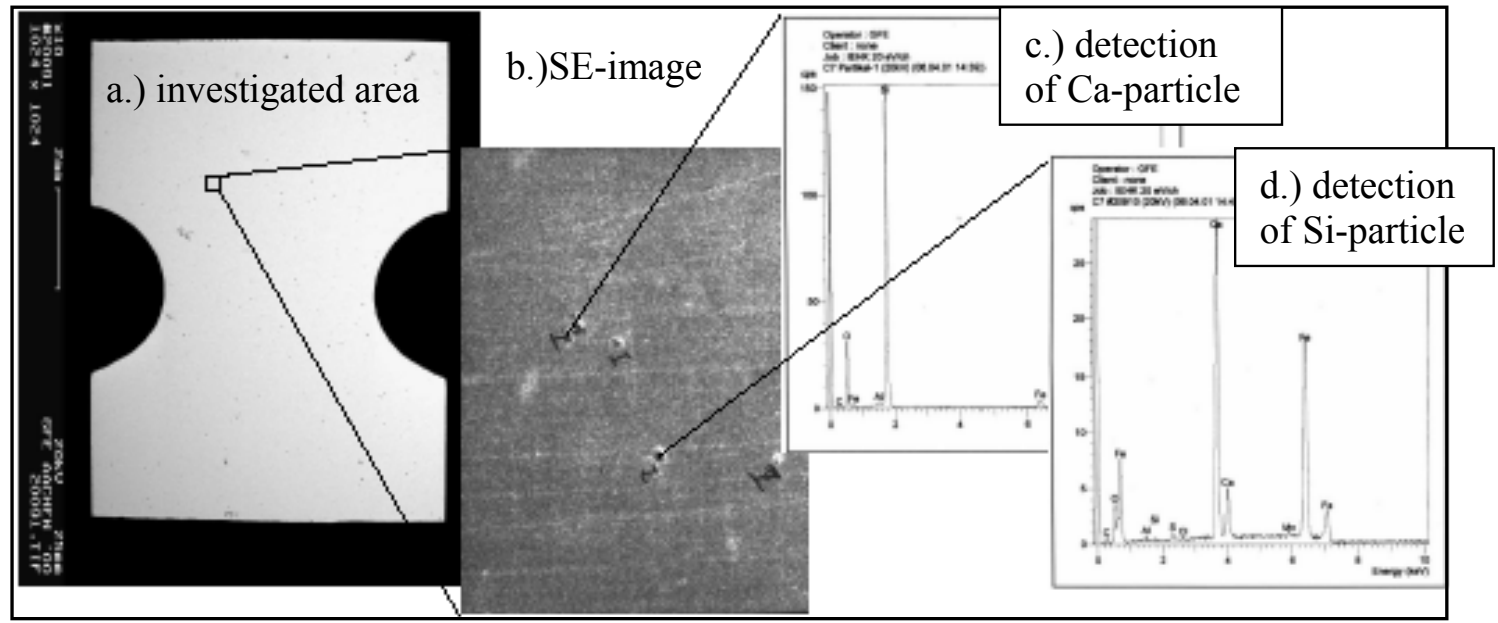

Figure 1: Detection of voids and inclusions using SEM and X-EDS, controlled by image analysis

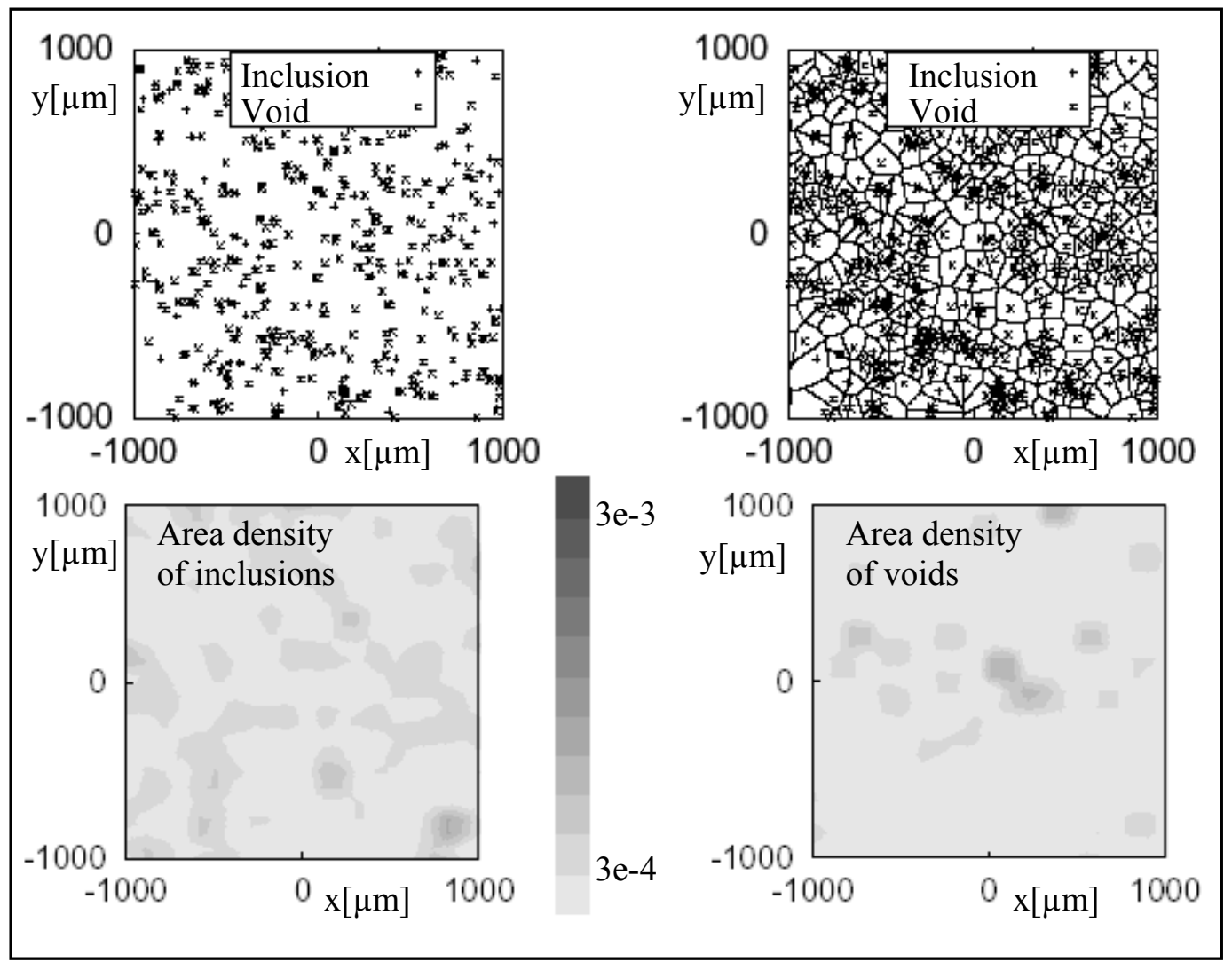

Figure 2: Determination of local area density of inclusions and voids using Voronoi-decomposition

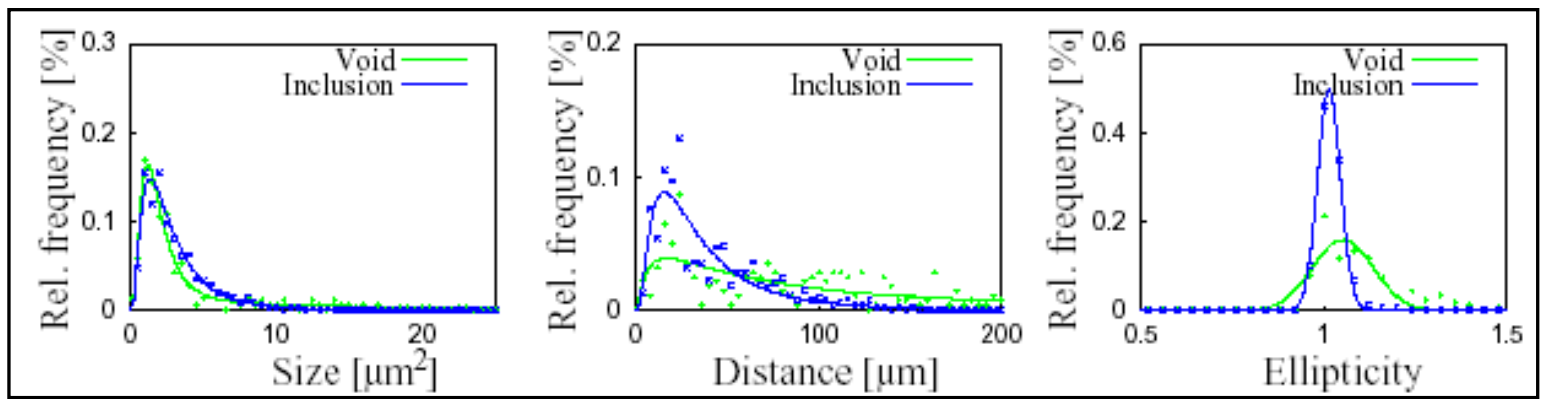

Figure 3: Microstructural characterisation of damage generating voids and inclusions in S469M 